\title{
EDUCACIÓN BASADA EN COMPETENCIAS: HACIA UNA PEDAGOGÍA SIN DICOTOMÍAS
}

\author{
JORGE ALARCón LEIVA* \\ BRIANNA HILL ${ }^{* *}$ \\ ClAUdio Frites $S^{* * *}$
}

\begin{abstract}
RESUMEN: El artículo examina una comprensión de la noción educativa de "competencias" desde una perspectiva que cabría designar como su justificación. Se abordan tres tópicos centrales. En primer lugar, se caracteriza la competencia lingüística como paradigma explicativo de la noción de "competencia". En segundo lugar, se ensayan tres acercamientos a la educación por competencias, a partir de una comparación con la pedagogía por objetivos, desde su evolución histórica y finalmente desde una perspectiva conceptual, dando cuenta de la necesidad de una pedagogía "sin dicotomías". Se concluye examinando algunas descripciones de la noción educativa de competencia para subrayar su comprensión como "inteligencia de la situación", con el fin de discutir sus sentidos y alcances apelando al marco de referencia conceptual derivado del examen de la competencia lingüística.
\end{abstract}

Palabras clave: Educación basada en competencias. Integración. Competencia lingüística. Gestión. Currículo.

\section{COMPETENCY-BASED EDUCATION:} FOR A PEDAGOGY WITHOUT DICHOTOMIES

\begin{abstract}
This paper proposes an examination of the conception of "competence" from a perspective that could be considered a justification for competency-based education. This work will address three main topics. First, it offers a description of linguistic competence as an explanatory paradigm of the notion of competence. Second, three different approaches towards competency-based education are examined, starting with a comparison with objective-based pedagogy, then from a historical perspective
\end{abstract}

\footnotetext{
* Instituto de Investigación y Desarrollo Educacional de la Universidad de Talca. Santiago de Chile - Chile.

** Programa de Idiomas de la Universidad de Talca. Santiago de Chile - Chile.

*** Instituto de Investigación y Desarrollo Educacional de la Universidad de Talca. Santiago de Chile - Chile.

Contato com os autores: <joalarcon@utalca.cl>
} 
and finally from a conceptual approach, taking into account the need for a Pedagogy "without dichotomies". Finally, several views about competence in education will be examined in order to highlight the conception as "understanding the situation", in an attempt to discuss their senses and scope, based on the conceptual framework derived from the examination of linguistic competence.

Key words: Competency-based education. Integration. Linguistic competence. Management. Curriculum.

\section{L'ENSEIGNEMENT FONDE SUR LES COMPETENCES: POUR UNE PEDAGOGIE SANS DICHOTOMIES}

RÉSUMÉ: Cet article propose une analyse de la notion de "compétence" sous une perspective qui peut être considérée comme une justification de l'enseignement fondé sur les compétences. D'abord, l'article considère trois sujets principaux. En premier, une description de la compétence linguistique comme un paradigme du concept de compétence. Ensuite, on examine trois approches vers l'enseignement fondé sur la compétence: une comparaison avec la pédagogie basée sur les objectifs, après une perspective historique et finalement une approche conceptuelle, en faisant le point sur les besoins de l'enseignement "sans dichotomies". Finalement on examinera plusieurs vues de l'enseignement par compétences pour mettre en évidence leur compréhension comme "l'intelligence de la situation", pour essayer de discuter sa signification et son ampleur, basé sur le cadre conceptuel dérivé de l'analyse de la compétence linguistique.

Mots-clés: L'enseignement fondé sur compétence. Intégration. Compétence linguistique. Gestion. Cursus universitaire.

\section{Introducción}

$\mathrm{E}$ 1 artículo aborda la idea de competencias tomando como ejemplo el examen de la competencia lingüística y el conjunto de desafíos pedagógicos implicados en ella. Para ello, se establece una relación entre la competencia lingüística, su desarrollo y cómo ésta puede utilizarse como modelo explicativo.

Luego, en consideración al ejemplo de la competencia lingüística, se señala la insuficiencia de los enfoques existentes para explicar el desarrollo de una competencia y la falsa suposición de la independencia entre método y contenido, que caracteriza a ciertos enfoques pedagógicos. Esta crítica se elabora a partir de la propuesta surgida del examen de la competencia lingüística y sus rasgos definitorios, esto es, su carácter normativo, público y constituyente, los cuales hasta ahora han sido sólo parcialmente recogidos por la literatura especializada.

En la segunda sección, se problematiza la distinción entre método y contenido y las consecuencias que plantea en lo pedagógico, de lo que daría cuenta el creciente 
cuestionamiento a la educación por objetivos. En este contexto, parte del éxito del concepto de competencias se sostiene que corresponde a que se ha hecho cargo del diagnóstico de un problema pedagógico crucial, aunque todavía resta por ofrecer una caracterización robusta sobre sus consecuencias para el proceso pedagógico. De ahí que el diseño curricular por competencias se encuentre aún sub-teorizado, pese a la profusa utilización del enfoque por competencias en el discurso educativo.

La dicotomía entre método y contenido se problematiza a partir de razones que atañen a la comprensión que suele estar a la base de muchos procesos formativos y forma parte del tipo de rasgo que resulta más difícil de comprender y que, no obstante, es el más prometedor de la educación basada en competencias. De este modo, se concluye, la Educación Basada en Competencias (EBC), así entendida, plantea la exigencia de una pedagogía sin dicotomías, en que la decisión metodológica y el contenido no están disociados lógica y temporalmente.

A continuación se analiza la evolución de la competencia desde una perspectiva histórica y conceptual, para señalar que hay una cierta convergencia entre ambas perspectivas, en cuanto la evolución del concepto de competencias describe un curso que lleva de considerarla como respuesta a una tarea hasta la constitución de la experiencia, pasando por seguir una regla. Complementariamente, se muestra y discute la evolución del concepto representada en autores tales como Perrenoud (2004), Jonnaert et al. (2004), Le Boterf (2000) y Tardif (2006), examinando descripciones de la noción educativa de competencia como "inteligencia de la situación"

\section{La competencia lingüística como técnica de uso normativa, pública y constituyente del lenguaje natural}

En esta sección se propone una explicación de la "competencia lingüística" que dé cuenta de su desarrollo como del aprendizaje de un sistema de reglas inherente a una práctica. Como resultado de este análisis, se obtiene una caracterización de los rasgos centrales del dominio lingüístico, en relación con su carácter normativo, social y constitutivo. Tal resultado, como podrá verse enseguida, se aplica prácticamente sin modificaciones a la comprensión de cualquier dominio de acción, vale decir, a cualquier competencia.

El entrenamiento en un lenguaje se presenta en contextos en los que prontamente emerge el problema de la normatividad, esto es, en situaciones en que es importante lograr un contraste sistemático entre usos correctos y usos incorrectos de palabras (WILLIAMS, 1999). En dichos contextos, son distintas las estrategias utilizadas para lograr el dominio de la competencia por parte del aprendiz y son distintas las formas en que tales estrategias pueden ser caracterizadas. El uso de 
estrategias para el desarrollo de la competencia lingüística revela, a su vez, los distintos roles que surgen en distintos contextos de desempeño.

En primer lugar, considérese el rol que emerge en el contexto de la distinción entre enseñanza y definición ostensiva (ibíd.). La cuestión se relaciona con la dificultad para aceptar que la definición ostensiva sea una explicación del fenómeno de adquisición del lenguaje por parte de un aprendiz, puesto que esa explicación implica una regresión al infinito, esto es, implica la necesidad de apelar a un nuevo fundamento una y otra vez, sin que el proceso de justificación pueda ser concluido nunca. El ataque a la definición ostensiva - a la noción de identidad, de miembro de una clase y de identificación de ese miembro por medio de la ostensión - revela importantes aspectos relacionados con el lenguaje y su adquisición: en primer lugar, supone abandonar la idea que la relación palabra-objeto sea central, que es una clave de bóveda de la concepción del lenguaje que se rechaza; en segundo lugar, que comprender el funcionamiento del lenguaje no puede tener lugar sin hacer referencia a un contexto (WILLIAMS, op. cit.).

La cuestión se resuelve mediante la sustitución de la noción de definición ostensiva por la de "entrenamiento ostensivo" (WITTGENSTEIN, 1988), el cual permite evitar la regresión al establecer el contexto de desempeño. Existe cierta afinidad entre la noción de un "entrenamiento ostensivo" y la idea conductista de "condicionamiento", pero se trata sólo de una afinidad, puesto que el entrenamiento se entiende como una práctica normativa.

El entrenamiento explica mejor la adquisición de un lenguaje por medio de la asociación conductualmente condicionada que resulta del ejercicio de repetir la misma actividad. El aprendiz, sin embargo, no es una tabula rasa: la enseñanza ostensiva requiere que él tenga ciertas capacidades conductuales y perceptivas; de otra manera podría fallar. Pero no se trata de que las más altas capacidades cognitivas estén posibilitadas por unas capacidades más bajas: lo que el aprendiz está haciendo es adaptar su conducta a normas, para lo cual el dominio del lenguaje es un medio indispensable. "Normativo", como aquí se entiende (WILLIAMS, 1998, p. 193), está restringido a actuaciones (performances), verbales y no verbales, que pueden ser juzgadas como correctas o incorrectas, e individuadas por referencia a normas, estándares, ejemplos o reglas.

Para abreviar, lo que se muestra es una competencia normativa que es manifestada en una práctica gobernada por reglas. El aprendiz no tiene esta competencia, sólo el que enseña o el que domina la práctica la tiene: al aprendiz le falta el background que sí tiene el que enseña (WILLIAMS, 1999) y dicho background sólo puede ser provisto por el medio social personificado - encarnado - en la acciones del maestro (WILLIAMS, op. cit.; VARELA, 2000; MASCIOTRA, 2002). 
En segundo lugar, el rol del aprendizaje permite revelar la fuente de la normatividad, al distinguir contextos de aprendizaje en los que actúa un aprendiz de aquellos en los que actúa un experto en la práctica, siendo aquí el principal problema cómo llega un novicio a seguir-una-regla, esto es, cómo una persona lingüísticamente incompetente llega a ser competente (MASCIOTRA, 2000).

Hay razones positivas para pensar que el contexto (stage setting) requerido para el aprendiz debe ser público, vale decir, social (WILLIAMS, 1999). Es la caracterización social del significado la que hace el trabajo de situar la actuación en el contexto apropiado. De ahí que algunos autores sugieran para la actuación la idea de "en acción" que permite justamente superar la dicotomía actuación-contexto (MASCIOTRA, 2000; VARELA, 2000).

Una consecuencia importante de ello es la indispensabilidad del entrenamiento inicial. Parafraseando a Quine, que el significado sea socialmente aprendido son las dos indispensables caras de la misma moneda. Si el significado es inherentemente social, entonces la hipótesis individualista del cognitivismo y de la teleología natural no pueden explicar lo que se proponen explicar, el primero por su mala interpretación de los conceptos de regla y representación, y el segundo porque no genera normatividad (WILLIAMS, 1999). De ahí que lo que se requiere es una concepción socio-genética de desarrollo (VARELA, 2000).

Finalmente, y en conexión directa con la cuestión aludida en la Introducción respecto a la falsa suposición de la independencia entre método y contenido, está el rol que contiene la idea de que cómo aprendemos conceptos es constitutivo de lo que aprendemos (WILLIAMS, 1999). Esta perspectiva se plantea contra la presunción de que la relación entre el aprendizaje y su producto es contingente, un asunto de "mera historia" (VARELA, 2000). No hay tal cosa como "el método de aprendizaje" de una parte y "el contenido aprendido" por otro, puesto que las prácticas de aprendizaje mantienen una relación intrínseca, es decir, no circunstancial, con lo que se aprende al llevarlas a cabo - decimos "[...] llevarlas a cabo" en vez de "[...] a través de ellas" para evitar inducir la distinción entre la práctica (método) y su objeto (contenido). La práctica, dicho de otra manera, se lleva a cabo porque tiene un contenido, sin que por ello se pueda decir que pudiera no tenerlo, por cuanto es esta práctica particular porque tiene este contenido específico; en general, es lo que se quiere decir con la expresión "intención" en la tradición fenomenológica.

En otras palabras, esta perspectiva se opone a la concepción que consiste en sostener que describir cómo adquirimos creencias - si mediante instrucción explícita, tomando alguna píldora apropiada, por osmosis o por accidente - es irrelevante para el contenido de tales creencias. Si cómo aprendemos es constitutivo de lo que aprendemos, entonces el aprendizaje juega un rol indispensable para comprender 
las creencias que tenemos. Insistamos en este punto: el método de aprendizaje constituye el contenido del aprendizaje, al punto que es plausible sostener la completa indivisibilidad entre uno y otro.

La discusión sobre el aprendizaje del lenguaje recalca que hay ciertos aspectos del dominio del lenguaje que enfoques conductistas y cognitivistas no pueden explicar. Crucialmente, tales enfoques no pueden explicar cómo la conducta de los aprendices llega a estar estructurada por normas; por normas o estándares de corrección que subyacen al uso del lenguaje. De acuerdo con ello, el aprendizaje del lenguaje es más que un agregado de disposiciones verbales o de hipótesis bien confirmadas; es, antes bien, un conjunto de estándares normativos para la aplicación de palabras - lo que se llama una "técnica de uso". La expresión "para la aplicación de palabras", no obstante, no debe generar la confusión concerniente a entender la aplicación como el momento en que se traspasan los estándares a la práctica: la práctica precisamente es así porque encarna los estándares normativos que la constituyen.

El dominio lingüístico es así el resultado del entrenamiento en una técnica de uso que es simultáneamente normativa, pública y constituyente, y son éstos precisamente los rasgos que definen una competencia en general. La competencia lingüística es una instancia ejemplar de competencia - un paradigma en sentido gramatical - porque ella muestra que si tiene el carácter de una competencia permite entender al mismo tiempo los rasgos básicos de toda competencia.

En este sentido, una competencia es normativa porque su realización (performance) es efectuada en un contexto en que es posible la corrección; que la corrección sea posible, no obstante, no es un elemento accesorio o adyacente a la realización de la competencia - a su dominio manifiesto -, sino que el que podamos hablar de "dominio de competencias", de "nivel de logro", de "hitos de desarrollo" y demás se halla condicionado por el carácter intrínsecamente normativo de la propia competencia.

\section{La resolución de la competencia-en-la-acción}

Las puntualizaciones del apartado anterior han sido expuestas con la finalidad de servir de marco de referencia para una discusión acerca de distintos modelos conceptuales de competencias.

\section{Pedagogía por objetivos y educación basada en competencias}

Una revisión de la literatura pedagógica de las últimas décadas revela el desarrollo de varias tentativas por definir un marco de referencia conceptual para las competencias, lo que ha suscitado una diversidad de connotaciones del concepto. ${ }^{1}$ 
En efecto, las propuestas de definición han generado una ambigüedad e imprecisión que dificulta la utilización del concepto de competencia en el diseño curricular (JONNAERT et al., 2004), en tanto que se exige que el punto de partida del diseño resida justamente en adoptar una noción compartida de competencia (TARDIF, 2003). De ambos puntos de vista deriva una paradoja: para diseñar curricula por competencias se requiere una noción de competencia; no obstante, se reconoce la falta de acuerdo acerca de una definición que satisfaga los requerimientos implícitos y las necesidades de orientación para la gestión del diseño, su implementación y evaluación.

Como explicación para el estado del arte de la cuestión curricular, se puede aplicar el modelo de "cambio de paradigma" propuesto por Kuhn (1969). El esquema explicativo de Kuhn permite hacer frente a las dificultades de la situación del currículo, en términos de la tensión entre tradición e innovación. En este sentido, dada la coexistencia de diseños curriculares por objetivos (PPO) y por competencias (EBC), puede constatarse que no existe un paradigma en educación; en efecto, en el ámbito del diseño curricular conviven lenguajes, prácticas y valores distintos.

Bajo esta perspectiva puede sostenerse que el diseño curricular se encuentra en un momento pre-paradigmático, siguiendo todavía a Kuhn, vale decir, la PPO orienta el diseño de la EBC en combinaciones inarticuladas, afrontando desafíos sub-teorizados y con efectos nocivos para la producción de aprendizajes. Debido a ello justamente es que se presentan tantas controversias y la sensación permanente de estar recién comenzando cada vez. La Tabla 1 reúne estas observaciones, comparando la PPO con la EBC.

En orden de presentación, la primera de las columnas de la tabla señala que desde la perspectiva epistemológica existe un contraste evidente entre la PPO y la $\mathrm{EBC}$, consistente en que mientras que la primera asienta su base en el conductismo, la segunda se funda en un enfoque socio-constructivo (TARDIF et al. 1992; JONNAERT et al., 2004).

Las implicaciones de esta diferencia permiten introducir una serie de otras distinciones, entre ellas la que se representa en la segunda columna referida al diseño curricular, que resalta que la PPO se estructura sobre la base de los contenidos disciplinarios, en tanto que la EBC lo hace con base en situaciones globales, que requieren el concurso interdisciplinar para abordarlas. Las dos columnas siguientes derivan de la segunda: en efecto, tanto la prioridad didáctica de los contenidos como el énfasis en la actividad del docente de la PPO son consecuencia de su enfoque didáctico centrado en contenidos disciplinares. Finalmente, la EBC está comprometida con una comprensión amplia y articulada de recursos que concurren al desempeño competente del aprendizaje en situaciones contextualizadas. 
Tabla 1

Comparación entre PPO y EBC

\begin{tabular}{|c|c|c|c|c|c|c|}
\hline $\begin{array}{l}\text { Enfoque } \\
\text { curricular }\end{array}$ & $\begin{array}{c}\text { Perspectiva } \\
\text { epistemológica }\end{array}$ & $\begin{array}{l}\text { Diseño } \\
\text { curricular }\end{array}$ & $\begin{array}{l}\text { Enfoque } \\
\text { didáctico }\end{array}$ & $\begin{array}{c}\text { Prácticas } \\
\text { pedagógicas }\end{array}$ & Recursos & $\begin{array}{c}\text { Lógica de } \\
\text { desarrollo } \\
\text { de programas } \\
\text { de estudios }\end{array}$ \\
\hline $\begin{array}{l}\text { Pedagogía } \\
\text { por } \\
\text { objetivos } \\
\text { (PPO) }\end{array}$ & Conductismo & $\begin{array}{l}\text { Recorte de los } \\
\text { contenidos de } \\
\text { aprendizaje en } \\
\text { micro- } \\
\text { objetivos }\end{array}$ & $\begin{array}{l}\text { Contenidos } \\
\text { disciplinarios }\end{array}$ & $\begin{array}{l}\text { Énfasis en } \\
\text { el docente }\end{array}$ & $\begin{array}{l}\text { Contenidos } \\
\text { disciplinarios, } \\
\text { aislados y } \\
\text { descontextuali- } \\
\text { zados }\end{array}$ & $\begin{array}{l}\text { Conocimiento } \\
\text { descrito en } \\
\text { el programa } \\
\text { y situaciones } \\
\text { como } \\
\text { pretextos }\end{array}$ \\
\hline $\begin{array}{l}\text { Educación } \\
\text { basada en } \\
\text { competencias } \\
\text { (EBC) }\end{array}$ & $\begin{array}{l}\text { Socio- } \\
\text { construc- } \\
\text { tivismo }\end{array}$ & $\begin{array}{l}\text { Abordaje por } \\
\text { situaciones } \\
\text { globales e } \\
\text { interdiscipli- } \\
\text { narias }\end{array}$ & $\begin{array}{l}\text { Situaciones } \\
\text { de formación }\end{array}$ & $\begin{array}{l}\text { Énfasis en } \\
\text { el estudiante }\end{array}$ & $\begin{array}{l}\text { Variedad } \\
\text { de recursos } \\
\text { contextualizados } \\
\text { en la situación } \\
\text { de formación }\end{array}$ & $\begin{array}{l}\text { Situación } \\
\text { en primer } \\
\text { lugar y el } \\
\text { conocimiento } \\
\text { como } \\
\text { recurso del } \\
\text { desempeño } \\
\text { competente }\end{array}$ \\
\hline
\end{tabular}

Fuente: Elaboración propia a partir de Jonnaert et al. (2006).

Conviene retomar aquí la cuestión de la indistinción entre método y contenido. Ahora desde la perspectiva del contraste entre la PPO y la EBC. Dicho contraste justifica el rechazo de una perspectiva dualista en prácticamente todas las dimensiones de la comparación contenida en la Tabla 1. Este rechazo puede argumentarse desde la perspectiva de la EBC, subrayando que ella busca cuando menos reducir y óptimamente eliminar dicotomías pedagógicamente infructuosas como conductas y pensamientos, conocimientos y competencias, contenidos conceptuales y aplicaciones prácticas y, por fin, entre planificación e implementación curricular.

Todo ello se consuma en la acción, concebida como bastante, más que la mera ejecución física de comportamientos observables posteriores al procesamiento cognitivo de información o que el sólo acto cognitivo de alcanzar comprensión antes de actuar. Que se diga que los procesos cognitivos son anteriores y los comportamientos posteriores son el resultado de la prioridad lógica y temporal que se concede a los procesos mentales, presunción que descansa en un supuesto discutido y discutible, del que no se dispone de evidencia definitiva. A fortiori, la EBC favorece una concepción de cómo desarrollar competencias que suponen una pedagogía sin las dicotomías que caracterizan a la PPO.

\section{Las competencias en perspectiva histórica}

Estas diferencias pueden verse también como una transición histórica como parte de la evolución - del concepto de competencia. Así lo ha propuesto, por 
ejemplo, Goudreau et al. (2009) quienes sugieren que el concepto de competencia ha evolucionado desde sus orígenes, al punto que el actual desarrollo corresponde a una segunda generación de la EBC. La primera se caracterizó por su énfasis en el aprendizaje a base de tareas (task-based learning), las cuales se asocian a funciones repetitivas enseñadas en la educación técnica; mientras que la segunda se centra en un modelo constructivista que abarca e involucra estrategias metodológicas relacionadas con el logro de competencias de alto nivel, típicamente desarrolladas en la educación superior (HAWES; CORVALÁN, 2006).

Esta distinción entre la primera y segunda generación de EBC también se puede entender en términos de la amplitud del enfoque. Hager y Gonzci (1996) comparan conceptos de competencia para indicar que la tendencia en el pasado era definir competencia de una manera estrecha, lo cual perjudicó a los beneficios potenciales del enfoque; mientras que actualmente, en países como Australia, la visión aplicada ha sido más amplia, la cual no sólo es más coherente con la lógica del concepto de competencia sino que también brinda más beneficios. ${ }^{2}$ En este contexto, los autores mencionan tres enfoques en orden creciente de amplitud: la lista de cotejo ("checklist" approach), el enfoque genérico (generic approach) y el integrado (integrated approach).

El primer enfoque, la lista de cotejo, coincide con la primera versión de EBC en que se concibe de competencia como la capacidad de desempeñar una serie de tareas discretas y observables, las cuales se podrán evaluar a través de una lista de descripciones de éstas, sin contemplar los aspectos más extensos del desempeño competente, por ejemplo la planificación o la reacción a imprevistos.

Los autores profundizan esta distinción entre los estrechos enfoques antiguos y el amplio enfoque nuevo a través de su tratamiento del tipo genérico. Se presenta éste como una respuesta a las limitaciones de la lista de cotejo, ya que se concibe de competencia como la posesión de un conjunto de atributos deseados, incluyendo conocimientos relevantes, habilidades y capacidades, como la resolución de problemas, análisis, comunicación, etc., y actitudes apropiadas. Aunque este enfoque parece más prometedor en el levantamiento de información sobre la variedad de aspectos no previsibles, fuera de la rutina del trabajo, se lo ha criticado porque se tratan los atributos en aislamiento del contexto del trabajo, el cual impide la transferencia de aprendizaje a situaciones profesionales reales.

El tercer y último enfoque identificado por Hager y Gonzci (op. cit.), el enfoque integrado, ya se sitúa el concepto de competencia en la denominada segunda generación de EBC, señalando que la clave está en la integración del contexto de situaciones profesionales. Asimismo, en el contexto de la segunda generación de EBC, una competencia es un saber-hacer basado en la combinación y movilización de conocimientos, habilidades, actitudes y recursos externos, aplicados apropiadamente a 
situaciones específicas, relacionadas con el contexto profesional del estudiante (TARDIF, 2006) de una manera holística y sistémica (GOUDREAU et al., 2009; HAGER; GONCZI, 1996).

La segunda generación implica el aprendizaje basado en problemas, el aprendizaje cooperativo y la pedagogía reflexiva (narrative pedagogy), entre otros, lo cual involucra la definición de las competencias profesionales asociadas con cada práctica de la profesión determinada. Esta puntualización histórica recoge su sentido de una distinción en su base que se supone que justifica distinguir entre dos tipos de acciones: por una parte, tareas; por otra, prácticas o acciones complejas. Esta distinción funciona a condición que se acepte que una tarea es más imple que una práctica.

En otras palabras, la justificación de la perspectiva histórica reseñada deriva de aceptar la dicotomía entre tarea y práctica, posible de sostener a condición de aceptarse que las tareas son simples y las prácticas son complejas. Ello implicaría conceder también que "simplicidad" y "complejidad" constituyen atributos ontológicamente relevantes que brindan validez a la diferencia que se arguye entre tareas y prácticas. Pero esto es nuevamente discutido y discutible a menos que a su vez se asuma como verdadero que las tareas son actividades no intencionales y las prácticas sí lo son, cuestión que por esta vía sería difícil establecer. Tareas y prácticas parecen más bien partes de un continuum a las que perfectamente puede reconocérseles intencionalidad, sin pérdida cognitiva.

\section{Las competencias en perspectiva conceptual}

Otra manera de avanzar no reside en proponer cierta manera de organizar la evolución del concepto de competencia, sino que mostrando una suerte de evolución conceptual "interna” a su desarrollo. Organizando, entonces, las definiciones de competencias concebidas como tareas, como la práctica normativa de seguir reglas (sociales o públicas) y como constitutiva de una experiencia situada. La diferencia entre los tres elementos de la evolución dependerá de aceptar que mientras las tareas no son intencionales, las prácticas lo son, aunque carecen de la articulación que deriva de su pertenencia a un dominio de prácticas, como ocurre con la experiencia situada.

Esta organización conceptual describe relativamente la misma propuesta contenida en el apartado acerca de competencia lingüística. Considerando esta manera de entender la noción de competencia, considérese el siguiente conjunto de definiciones, contenidas en la Tabla 2.

En la Tabla 2 se recogen una serie de cuatro definiciones de competencias, ofrecidas por autores de referencia usual en el contexto del debate chileno, en las que se combinan aspectos que hacen más sensible la definición a los rasgos que han permitido su caracterización como tarea, seguir reglas y como constitutiva de una 
experiencia. Así por ejemplo, la propuesta de Perrenoud (2004) no calificaría para ser considerada una "competencia", puesto que está comprometida con un concepto disposicional de "capacidad" como atributo, que resulta elusivo respecto del carácter "activo", de "ejecución efectiva", "performativo" de la competencia. Adicionalmente, se trata de una concepción limitadamente cognitiva en su condición y, en fin, que acude a la consciencia como criterio de realización.

\section{Tabla 2}

Descripción de definiciones del concepto de competencia

\begin{tabular}{|c|c|c|c|c|c|c|}
\hline Autor & Atributo & Acción & Constructo & Condición & Contexto & Criterio \\
\hline $\begin{array}{l}\text { Perrenoud } \\
(2004)\end{array}$ & "capacidad" & $\begin{array}{l}\text { determinar } \\
\text { y realizar }\end{array}$ & $\begin{array}{l}\text { una acción } \\
\text { adaptada a } \\
\text { la situación }\end{array}$ & $\begin{array}{l}\text { movilizando } \\
\text { "varios recursos } \\
\text { cognitivos" }\end{array}$ & $\begin{array}{l}\text { en "un tipo } \\
\text { de situaciones" }\end{array}$ & $\begin{array}{l}\text { determinar } \\
\text { "de un modo } \\
\text { consiente y } \\
\text { rápido" y } \\
\text { realizar "de un } \\
\text { modo eficaz" } \\
\text { una acción }\end{array}$ \\
\hline $\begin{array}{l}\text { Jonnaert } \\
\text { et al. (2004) }\end{array}$ & $\begin{array}{l}\text { "puesta } \\
\text { en marcha" }\end{array}$ & permitir & $\begin{array}{l}\text { el "tratamiento" } \\
\text { de una situación }\end{array}$ & $\begin{array}{l}\text { eligiendo, } \\
\text { movilizando } \\
\text { y organizando } \\
\text { un "conjunto } \\
\text { diversificado } \\
\text { y coordinado } \\
\text { de recursos" }\end{array}$ & $\begin{array}{l}\text { en "un contexto } \\
\text { determinado" }\end{array}$ & con éxito \\
\hline $\begin{array}{l}\text { Le Boterf } \\
(2000)\end{array}$ & $\begin{array}{l}\text { "saber } \\
\text { actuar" }\end{array}$ & lograr & "un resultado" & $\begin{array}{l}\text { "combinando } \\
\text { y movilizando } \\
\text { recursos } \\
\text { necesarios" }\end{array}$ & $\begin{array}{l}\text { en "un contexto } \\
\text { de trabajo" }\end{array}$ & $\begin{array}{l}\text { con un } \\
\text { resultado } \\
\text { "excelente } \\
\text { y validado } \\
\text { en una } \\
\text { situación } \\
\text { de trabajo" }\end{array}$ \\
\hline $\begin{array}{l}\text { Tardif } \\
(\mathbf{2 0 0 6 )}\end{array}$ & $\begin{array}{l}\text { "saber } \\
\text { hacer } \\
\text { complejo" }\end{array}$ & actuar & & $\begin{array}{l}\text { movilizando } \\
\text { y combinando } \\
\text { "variedad de } \\
\text { recursos internos } \\
\text { y externos" }\end{array}$ & $\begin{array}{l}\text { "dentro de } \\
\text { una familia } \\
\text { de situaciones" }\end{array}$ & eficazmente \\
\hline
\end{tabular}

Fuente: Elaboración propia a partir de las referencias señaladas en cada caso.

Jonnaert et al. (2004), aunque alineada con una concepción más completa de competencia, proponen una definición que resulta innecesariamente figurativa, poco descriptiva, por lo tanto, cuando considera al atributo como una "puesta en marcha", imprecisa cuando al referirse a la condición se alude a una "variedad de recursos" sin más especificación y estrecha en su alcance cuando remite a un "contexto de trabajo". No obstante ello, como podrá constatarse más adelante, existe una reformulación del punto de vista de Jonnaert et al. (op. cit.) examinado 
aquí que permite percibir una importante serie de cambios que se ajustan más efectivamente con la comprensión de competencias que interesa subrayar. En estas reformulaciones desaparecen la metáfora, la imprecisión y la estrechez del alcance, dando pie a una comprensión de competencia más sensible a la situación-de-acción.

Le Boterf (2000) y Tardif (2006) subrayan la condición activa de la competencia, al referirse a la acción como "saber actuar" y "saber actuar complejo" respectivamente. Esta manera de entender la acción competente sugiere que no se trata de un "mero hacer", sino de un "hacer con saber", lo que en el caso de Tardif se intenta sofisticar mediante la introducción de "complejo" como cualificación de la acción. Ambas definiciones, además, consideran la referencia a "recursos" que deben ser "movilizados y combinados", aunque en el caso de Tardif se precisa que se trata de recursos "internos y externos". Esto último quiere decir que los recursos necesarios para afrontar el contexto en que tiene lugar la competencia conciernen a las destrezas disponibles para el aprendiz, tanto si son propias del sujeto como si pertenecen al entorno de su desempeño.

Por otra parte, la propuesta de Tardif introduce la importante referencia a una "familia de situaciones". Esta idea tiene un valor generativo en la discusión sobre el contexto del desempeño que ha resultado altamente inspiradora. Nótese que se trata no de "una" situación (en singular) ni de una "serie" de situaciones (en plural) como una forma de garantizar la propiedad de la selección del contexto de acción competente. Es decir, no se trata ni de distinguir la acción en términos de mayor o menor complejidad, ni de diferenciarla en cuanto al número de situaciones que lograrían saturar la competencia. La estrategia supone más bien indicar que en un amplio rango existen situaciones que tienen semejanzas entre sí, como la semejanza que presentan dos individuos - dos hermanos, pongamos por caso - pertenecientes a un mismo grupo familiar, y que aunque no se disponga siempre de límites fijos sobre los cuales basar una distinción fuerte, sí se puede establecer un "parecido" entre ellas (WITTGENSTEIN, 1988).

Este parecido no hace que las situaciones sean numéricamente iguales, pero permite decir que pertenecen al mismo grupo, tal y como Rosch $(1973,1978)$ ha sugerido que se establecen conceptos en su teoría de prototipos.

\section{Examinando descripciones de competencia: inteligencia de la si- tuación}

La referencia a la teoría de Rosch (op. cit.) es apropiada en cuanto permite entender cómo las situaciones se asemejan entre sí y cuánto depende de la capacidad del agente determinar el punto en que la situación deja o no de ser representativa del 
"prototipo" de situación al que están referidas. Se puede sugerir que ello deriva de cierta "inteligencia" del agente para apreciar la frecuencia en que ciertos rasgos se presentan para juzgar que la situación es "de la misma familia". Es decir, esta apreciación depende de la aplicación de un criterio, de una cierta regla. Lo que conduce a la consideración del modo de entender la competencia que constituiría una suerte de transición entre la concepción de la competencia como tarea y la concepción de la competencia como constitutiva de la experiencia.

Si consideramos al ser humano sólo como un tipo de organismo, desde una perspectiva naturalista, se le exigen adaptaciones más complejas, más rápidas y más complicadas que a ningún otro organismo vivo. El comportamiento humano, para satisfacer las exigencias cambiantes de un medio en constante cambio, tiene que ser flexible. Por esta razón, el organismo humano debe ser adaptable por medio de respuestas variables. La diferencia entre los seres humanos y otros organismos reside sólo en su más alto nivel de complejidad, lo cual concuerda con la imagen de la vida humana como resultado de procesos evolutivos filo y ontogenéticos.

La aptitud para elaborar tales series de comportamientos y aplicarlos de una manera oportuna al entorno, en el caso de los seres humanos, es el resultado tanto de procesos de desarrollo biológico como de aprendizaje en un contexto socio-cultural. La relación entre desarrollo y aprendizaje, entendidos de la manera recién descrita, constituye un punto de partida mediante el cual ponderar las cualidades específicamente humanas de la maduración biológica. Permite considerar que el aprendizaje humano, a diferencia del "aprendizaje" de otros seres, tiene que explicar la formación de creencias y el hecho de que tales creencias forman una totalidad ligada a las intenciones y propósitos del agente.

La variabilidad y la flexibilidad para actuar y responder se adquieren mediante un proceso de aprendizaje sujeto a patrones normativos de comportamiento, los cuales aparecen junto con la capacidad de aprender a hacer uso del lenguaje. Mediante sus intercambios con el medio, el organismo humano desarrolla la aptitud para elegir comportamiento más adecuados, y dicha aptitud no puede comprenderse en los términos puramente descriptivos de las ciencias naturales, sino que también mediante el uso de expresiones que den cuenta de la relación entre creencias y acciones (actitudes proposicionales). Expresiones mediante las cuales atribuimos al agente creencias e intenciones; verbos psicológicos tales como "pensar", "desear", "tener la intención", "esperar", entre otros, constituyen parte de la forma habitual mediante la cual nos explicamos, conforme a un patrón explicativo cotidiano, la racionalidad de un agente humano.

Lograr un practicante normativo cuyas acciones estén guiadas por reglas requiere regularizar la práctica del novicio. Sin normatividad, la noción de "hacer lo 
mismo" se vuelve vacía; y juzgar "lo mismo" es esencial para ser guiado por reglas o normas. Es aquí el lugar en el cual se introduce la distinción entre modos de normatividad:

\section{Tabla 3}

Modos de comprender la forma de seguir reglas

\begin{tabular}{|ll}
\hline Conducta que se conforma a reglas & $\begin{array}{l}\text { La conducta se conforma como materia de hecho al patrón, } \\
\text { sin que éste forme parte de la acción sino que es su } \\
\text { referencia. }\end{array}$ \\
\hline Conducta que obedece reglas & $\begin{array}{l}\text { Consiste en comportarse con la intención de realizar un } \\
\text { sistema de movimientos o con la intención de seguir reglas } \\
\text { o un patrón, sin que ello implique seguir efectivamente la } \\
\text { regla. }\end{array}$ \\
$\begin{array}{l}\text { Conducta que es gobernada } \\
\text { por un patrón }\end{array}$ & $\begin{array}{l}\text { La distinción anterior escondía un tercer tipo de } \\
\text { regularidad, la conducta que ejemplifica un patrón } \\
\text { abstracto o una parte de ese patrón. }\end{array}$
\end{tabular}

Es esta última forma la que interesa, por cuanto es aquella de acuerdo con la cual la constitución de la experiencia no es puro arbitrio subjetivo, sino una sujeción a normas que es ejemplificado en la acción. En este sentido es una concepción que conduce a la resolución en la acción de la competencia. Jonnaert y su equipo de investigadores, en base a su análisis bibliográfico y sus experiencias prácticas, proponen una perspectiva contextualizada para el desarrollo de competencias, identificando tres pilares: 1) las situaciones, 2) el desempeño competente y 3) la inteligencia de las situaciones. Para aclarar el concepto contextualizado de las competencias, es importante referir a la noción "cognición situada" (JONNAERT et al., 2005, p. 684), entendiendo la cognición como parte de la práctica social y "distribuida sobre el cuerpo y la actividad de la persona en situación, sobre la situación en sí misma y sobre su contexto" (JONNAERT et al., 2006, p. 10). Así, la cognición se encuentra en el núcleo de un:

[C]onjunto de relaciones dialécticas entre la persona en acción, su propia cognición, la situación, sus contextos sociales y físicos. La persona actuante no está separada de su contexto de acción en el mundo, lo determina, a la vez que está determinada por él. La persona es un todo actuante, inmersa en el mundo, en un campo complejo de inter-relaciones: person-acting-in-setting. (LAVE, 1988 en JONNAERT et al., 2006, p. 10)

La "cognición situada" da una visión clara de la importancia de la persona, que se construye por medio de la construcción de su entorno, en relación dialéctica con él, para el desarrollo de sus competencias (JONNAERT et al., 2006, p. 10). 


\section{Conclusiones}

La muy amplia serie de cuestiones programáticamente elaboradas en el artículo permite obtener un importante número de consideraciones conclusivas, sobre las cuales convendrá trabajar sucesivamente tal vez por separado.

En primer lugar, se ha propuesto establecer una conexión explícita entre la noción misma de competencia, su desarrollo desde una perspectiva pedagógica y la referencia a ella como un modelo explicativo tanto de su propio desarrollo como de la pedagogía que fundamenta. La síntesis de ello se verifica en la idea de que el uso del concepto de competencia debiera permitirnos avanzar hacia una pedagogía sin dicotomías.

El modo de argumentar este primer punto de vista del artículo ha requerido comenzar caracterizando una forma de competencia que tiene un carácter paradigmático, a saber, la competencia lingüística. En particular, el desarrollo de la competencia lingüística muestra una primera consideración relevante de la competencia en general: su carácter normativo, público y constituyente. Este carácter es el que conduce a pensar la indistinción entre método y contenido, por cuanto justifica representarse el desarrollo de la competencia como una práctica social.

En segundo lugar, se puede concluir que una parte de la tarea pedagógica derivada de la adopción de la EBC permite elaborar un diagnóstico crítico sobre la PPO. Pero ello no puede ser todo porque se requiere proponer una perspectiva constructiva que permita entender la medida en que la EBC reduciría o, eventualmente, permitiría eliminar del todo el dualismo que caracteriza a la PPO.

Expresado raudamente, en este punto el artículo ofrece razones que justifican la consideración de la EBC como una pedagogía que obliga a pensar el método para desarrollar competencias en consonancia con las competencias a desarrollar. Sobre esta base, la decisión metodológica - último reducto del dualismo pedagógico - no puede distinguirse del contenido sino a riesgo de perder el carácter integral a que toda educación aspira. En consecuencia, no hay prioridad ni del método ni del contenido ni lógica, es decir, conceptualmente, ni temporalmente, es decir, en el orden práctico.

En tercer lugar, junto con examinar la evolución histórica y conceptual de las competencias, se puede señalar que ambas convergen allí donde finalmente una competencia se concibe como la manifestación de una regla, como un ejemplar, como un paradigma, de acuerdo con el cual el actuar competente no depende sólo de llevar a cabo acciones qua tareas, sino como una verdadera y real forma de constituir una experiencia.

Esto es lo que se ha querido subrayar con la noción de "inteligencia de la situación". Esta noción permite acceder a la idea cabal de competencia, puesto que 
permite expresar la relación intrínseca entre interior y exterior, es decir, entre inteligencia y situación, en virtud de la que no se puede hablar de la una sin la otra e inversamente. Una relación que coincide con desarrollos recientes en las así llamadas "ciencias cognitivas".

\section{Notas}

1. La preferencia por considerar a las "competencias profesionales" como tópico central de la educación basada en competencias es intencionada y se justifica porque se considera a éstas cuando menos en el debate nacional chileno - como las que conjugan en su formulación más densamente el amplio registro semántico en juego y sus diferencias.

2. Hager y Gonzci analizan las definiciones de "competence" y "competency" publicadas en distintos diccionarios para concluir que el concepto de competencia es relacional, es decir, vincula dos elementos distintos: los atributos de la persona y su completación satisfactoria de una tarea. Explican que es necesario tener una combinación de atributos y tareas para captar el concepto de desempeño competente, ya que "competence" se centra en habilidad o capacidad; y esta habilidad está compuesto por atributos, por un lado, mientras que estas apuntan a una tarea(s), por otro lado.

\section{Referencias}

GONCZI, A. Reconceptualising competency-based education and training: with particular reference for occupations in Australia. Sydney: University of Technology, 1996. Disponível em: <http://epress.lib.uts.edu.au/research/bitstream/ handle/10453/20143/01front.pdf?sequence=1>. Acesso em: 25 jun. 2013.

GOUDREAU, J. et al. A second generation of the competency-based approach to nursing education. International Journal of Nursing Education Scholarship, v. 6, n. 1, art. 15, April 2009.

HAGER, P.; GONZCI, A. What is competence? Medical Teacher, Basingstoke, v. 18, n. 1, p. 1-8, 1996.

HAWES, G.; CORVALÁN, O. Aplicación del enfoque de competencias en la construcción curricular de la Universidad de Talca, Chile. Revista Iberoamericana de Educación, Madrid, n. 40, 2006. Disponível em: < http://www.rieoei.org/ deloslectores/1463Corvalan.pdf $>$. Acesso em: 25 jun. 2013.

JONNAERT, P. et al. Contribution critique au développement des programmes d'études: compétences, constructivisme et interdisciplinarité. Revue des Sciences de l'Éducation, Montréal, v. 30, n. 3, p. 667-696, 2004.

JONNAERT, P. et al. Contribution critique au développement des programmes d'études: compétences, constructivisme et interdisciplinarité. Revista de Ciencias de la Educación, n. 3, p. 667-696, 2005. 
JONNAERT P. et al. Revisión de la competencia como organizadora de los programas de formación: hacia un desempeño competente. Montreal: Universidad de Québec; ORE, 2006. Disponível em: <http://www.ibe.unesco.org/fileadmin/user_upload/COPs/ Pages_documents/Competencies/ORE_Spanish.pdf>. Acesso em: 25 jun. 2013.

KUHN, T. La estructura de las revoluciones científicas. Santiago de Chile: FCE, 1969.

LAVE, J. Cognition in practice: mind, mathematics and culture in everyday life. Cambridge: Cambridge University, 1988.

LE BOTERF, G. Ingeniería de competencias. Barcelona: Gestión, 2000.

MASCIOTRA. La compétence comme pouvoir adaptatif aux situations nouvelles. 2000. Disponível em: <http://academia.edu/1116760/La_competence_comme_pouvoir_ adaptatif_aux_situations_nouvelles>. Acesso em: 25 jun. 2013.

MASCIOTRA, M. La pensée-en-acte: une compétence tout en action. 2002. Disponível em: $<$ http://www.academia.edu/1483133/La_pensee-en-acte_une_competence_tout_en_ action> Acesso em: 25 jun. 2013.

PERRENOUD, P. Diez nuevas competencias para enseñar: invitación al viaje. Barcelona: Graó, 2004.

$\mathrm{ROSCH}, \mathrm{E}$. On the internal structure of perceptual and semantic categories. In: MOORE, T. (Ed.). Cognitive development and the acquisition of language. New York: Academic, 1973. p. 111-144.

ROSCH, E.; LLOYD, B. (Ed.). Cognition and categorization. Hillsdale: Lawrence Erlbaum, 1978.

TARDIF, J. Développer un programme par compétences: de l'intention à la mise en oeuvre. Pédagogie Collégiale, Montréal, v. 16, n. 3, p. 36-44, mars 2003.

TARDIF, J. L'évaluation des compétences: documenter le parcours de développement. Montréal: Chenelière Education, 2006.

TARDIF, J. et al. Le développement des compétences: cadres conceptuels pour l'enseignement professionnel. Pédagogie Collégiale, Montréal, v. 6, n. 2, p. 14-19, déc. 1992.

VARELA, F.; SÁEZ, J.C. (Ed.). El fenómeno de la vida. Santiago de Chile: Comunicaciones Noreste, 2000.

WILLIAMS, M. Epistemological realism and the basis of scepticism. Mind (New series), Oxford, n. 97, p. 415-439, 1988. 
Educación basada en competencias: hacia una pedagogía sin dicotomías

WILLIAMS, M. Wittgenstein, mind and meaning: toward a social conception of mind. London: Routledge, 1999.

WITTGENSTEIN, L. Investigaciones filosóficas. Trad. A. García Suárez e U. Moulines. México, DF: Unam, 1988.

Recebido em 9 de julho de 2013.

Aprovado em 4 de abril de 2014. 